\title{
IncTUG1/miR-144-3p affect the radiosensitivity of esophageal squamous cell carcinoma by competitively regulating
} c-MET

Pan Wang ${ }^{1}$, Zhuanbo Yang ${ }^{2}$, Ting Ye ${ }^{3,4}$, Fei Shao ${ }^{1,3,4}$, Jiagen $\mathrm{Li}^{1}$, Nan Sun ${ }^{1 *}$ and Jie He $\mathrm{1}^{\text {}^{*}}$

\begin{abstract}
Background: Long noncoding RNAs (IncRNAs) are involved in the progression of various cancers and affect the response to radiotherapy. This study focused on clarifying the underlying mechanism by which IncTUG1 affects the radiosensitivity of esophageal squamous cell carcinoma (ESCC).

Methods: InCTUG1, miR-144-3p and MET expression levels were detected in ESCC tissues and cells by qRT-PCR. Western blotting was used to examine the protein levels of MET, p-AKT and EGFR. The dual-luciferase reporter system and RNA immunoprecipitation (RIP) assays were used to confirm the interaction between IncTUG1 and miR144-3p or miR-144-3p and MET. MTT, colony formation and flow cytometry assays were applied to examine the behavioral changes in EC9706 and KYSE30 cells.

Results: IncTUG1 was upregulated in ESCC cells and tissues, and IncTUG1 expression was associated with an advanced pathological stage. The bioinformatics analysis revealed that IncTUG1 could specifically bind to miR-144-3p, which was downregulated in ESCC. There was a negative correlation between InCTUG1 and miR-144-3p. LncTUG1 inhibition retarded proliferation and colony formation and induced apoptosis in ESCC cells. Moreover, IncTUG1 knockdown dramatically improved the effect of radiotherapy on ESCC development both in vivo and in vitro. Furthermore, MET was revealed as a downstream target of miR-144-3p and is downregulated by it. LncTUG1 promoted the progression of ESCC and elevated radiotherapy resistance in ESCC cells, accompanied by a high level of MET expression. Moreover, we found that knockdown of InCTUG1 enhanced the radiosensitivity of ESCC cells via the p-AKT signaling pathway.

Conclusion: Our results indicate that IncTUG1 enhances the radiotherapy resistance of ESCC by lowering the miR-144$3 p$ level and modulating the MET/EGFR/AKT axis.
\end{abstract}

Keywords: IncTUG1, miR-144-3p, MET, Radiosensitivity, Esophageal squamous cell carcinoma

\section{Background}

Esophageal carcinoma ranks 9th among the most lethal cancers and widely exists in the world. According to statistics, esophageal carcinoma is responsible for hundreds of thousands of deaths [1, 2]. Esophageal squamous cell carcinoma (ESCC) is the predominant histological subtype, accounting for $90 \%$ of all cases. As a high aggressive

\footnotetext{
*Correspondence: sunnan@vip.126.com; prof.jiehe@gmail.com

'Department of Thoracic Surgery, National Cancer Center/National Clinical Research Center for Cancer/Cancer Hospital, Chinese Academy of Medical Sciences and Peking Union Medical College, No.17 Panjiayuannanli, Beijing 100021, China

Full list of author information is available at the end of the article
}

malignancy, ESCC always accompanies a miserable clinical outcome [3]. Despite noteworthy advances in cancer diagnosis and therapy, the clinical outlook of ESCC patients remains dismal, with a five-year survival rate of less than $30 \%$ $[4,5]$. To date, traditional surgery remains the preferred treatment for patients with early ESCC, but for patients with advanced ESCC, chemotherapy or radiotherapy is used [6]. However, there are quite a few patients who do not benefit from single radiotherapy or obtain an ideal response [7]. Thus, there is an urgent need to find a potential biological marker to indicate radiosensitivity and guide radiotherapy in ESCC patients. 
Recently, long noncoding RNAs (lncRNAs) have been described as noncoding RNAs that participate in many cancers and affect the progression of tumors $[8,9]$. lncRNAs, long RNAs > 200 nucleotides (nt) in length without any detectable open reading frames, regulate distinct biological processes in cancer cells by sponging microRNAs (miRNAs) or impacting the functions of related proteins [10]. Previous studies have shown that a high level of lncTUG1 accelerates cell growth by silencing KLF2 in hepatocellular carcinoma [11]. Similar to oncogenic factors, the role of lncTUG1 in ESCC is to promote the proliferation and migration of ESCC [12]. Moreover, lncRNAs have been found to influence radiosensitivity by various mechanisms, including DNA damage repair, epithelial-mesenchymal transition (EMT), apoptosis, and autophagy [13]. For instance, lncFAM201 regulates the radiosensitivity of non-small cell lung cancer (NSCLC) by the EGFR/miR-370 axis [14]. However, whether lncTUG1 is involved in the regulation of the radiosensitivity of ESCC remains uncharacterized.

Numerous miRNAs affect many human diseases, especially cancers [15]. miRNAs are noncoding RNAs 20-25 nt in length that bind to the $3^{\prime}$ untranslated region ( $3^{\prime}-\mathrm{UTR}$ ) of a specific mRNA, resulting in degradation of the target mRNA or repression of the mRNA expression $[16,17]$. Many miRNAs have been verified to be related to anticancer treatments, including radiotherapy [18]. For instance, miR-145 regulates radiotherapy resistance by affecting the P53 signaling pathway in colorectal cancer [19]. At present, the important role of miR-144-3p as a tumor suppressor in cancer is uncovered [20, 21]; however, whether miR-144-3p acts as a radiosensitivity-related factor in ESCC cell lines and tissues needs to be investigated.

$\mathrm{C}$-MET is a receptor tyrosine kinase and activates a wide range of different cellular signaling pathways after binding to its ligand, hepatocyte growth factor [22]. MET is always associated with EGFR and can upregulate EGFR to increase the phosphorylation of AKT (p-AKT) [23]. As a key factor related to radiosensitivity, a high level of AKT phosphorylation usually reflects a resistance effect on cancer radiotherapy [24, 25]. Thus, it is critical to reduce the $\mathrm{p}$-AKT level to improve the benefits of cancer radiation therapy.

The aim of this study was to discover mechanisms that can enhance the response of ESCC to radiotherapy. Through bioinformatics analysis, we found that the lncRNA TUG1 may be involved in regulating the radiosensitivity of ESCC, and the role of lncTUG1 in ESCC was subsequently examined. These findings suggest that lncTUG1 enhances the radiotherapy resistance of ESCC by lowering the miR-144-3p level and modulating the MET/EGFR/AKT axis. Therefore, lncTUG1 provides a new possible theoretical basis for radiotherapy in ESCC and has become a potential therapeutic target.

\section{Methods}

\section{Clinical samples}

A total of 50 paired tumor and adjacent normal tissues were retrospectively collected from 50 patients with ESCC. All of the patients had primary, nondistant metastatic ESCC and had undergone complete surgical resection (esophagectomy) at the Cancer Hospital of the Chinese Academy of Medical Sciences (CAMS) between December 2014 and December 2018 after providing informed written consent and agreement. None of the patients received chemo- or radiotherapy prior to surgery. According to the National Comprehensive Cancer Network esophageal cancer guidelines, the normal tissues were at least $5 \mathrm{~cm}$ away from the primary lesions. All samples were stored at $-80^{\circ} \mathrm{C}$ before further processing. This study was approved by the Medical Ethics Committee of the Cancer Hospital of the CAMS. The clinical characteristics of the patients are shown in Table 1.

\section{Bioinformatics analysis}

Radiation sensitive and resistant samples were retrieved from Gene Expression Omnibus (GEO) repository (GSE61816 and GSE61772). Probes were annotated by

Table 1 The relationships between TUG1 expression level and clinicopathological characteristics of patients with ESCC

\begin{tabular}{|c|c|c|c|}
\hline \multirow[t]{2}{*}{ Characteristics } & \multicolumn{2}{|l|}{ Expression of } & \multirow[t]{2}{*}{$P$ value } \\
\hline & $\operatorname{Low}(n=25)$ & $\operatorname{High}(n=25)$ & \\
\hline Sex & & & 0.248 \\
\hline Male & 17 & 13 & \\
\hline Female & 8 & 12 & \\
\hline Age & & & $0.009^{*}$ \\
\hline$\leq 60$ & 14 & 5 & \\
\hline$>60$ & 11 & 20 & \\
\hline Tumor size (cm) & & & $0.001^{*}$ \\
\hline$\leq 5$ & 15 & 6 & \\
\hline$>5$ & 10 & 19 & \\
\hline Lymph node metastasis & & & $0.045^{*}$ \\
\hline Yes & 11 & 18 & \\
\hline No & 14 & 7 & \\
\hline Pathological Staging & & & 0.152 \\
\hline$I+\|$ & 13 & 8 & \\
\hline$I I I+I V$ & 12 & 17 & \\
\hline Smoking status & & & 0.239 \\
\hline Ever/current & 14 & 18 & \\
\hline Never & 11 & 7 & \\
\hline Alcohol consumption & & & 0.771 \\
\hline Ever/current & 16 & 15 & \\
\hline Never & 9 & 10 & \\
\hline
\end{tabular}

Low/high by the sample mean. Pearson $X 2$ test. ${ }^{*} P<0.05$ was considered statistically significant 
the platform information stored in GEO. For gene with multiple probes, the expression value was calculated by averaging the expression values of its probes. To make data from different dastset comparable, the ComBat algorithm implemented in R package sva were used to adjust the batch effects and the batch were set as the different GEO series. R package limma was used to identify the differential expressed genes (DEGs). The design model were generated by "model.matrix $(\sim 0+$ Resistance/Sensitive)".

\section{Cell culture}

Human esophageal epithelial cells (Het-1A) and ESCC cell lines (TE-13, KYSE140, EC9706, and KYSE30) were purchased from the Cell Bank of Type Culture Collection of Chinese Academy of Sciences (Shanghai, China) and cultured in RPMI 1640 medium supplemented with $10 \%$ fetal bovine serum (Gibco, USA) in a $37^{\circ} \mathrm{C}$ incubator with $5 \% \mathrm{CO}_{2}$.

\section{Quantitative real-time PCR (qRT-PCR)}

Total RNA was extracted with TRIzol reagent (Invitrogen, Carlsbad, CA, USA). cDNAs were synthesized with a reverse transcription kit (Invitrogen). qRT-PCR analysis was performed with SYBR Premix Ex Taq II (TaKaRa, Dalian, China). For mRNA and miRNA, GAPDH and U6 were used as internal controls, respectively. The primers are shown in Table 2.

\section{Cell transfection}

After reaching $40-50 \%$ confluence, cells were transfected with a small interfering RNA (siRNA) targeting TUG1 (si-TUG1), a miR-144-3p mimic, a miR-144-3p inhibitor, si-MET, LV-TUG1 and a nonspecific control (Invitrogen, Shanghai, China) by using Lipofectamine 3000 (Invitrogen, USA).

Table 2 The sequences of specific primers

\begin{tabular}{ll}
\hline Gene name & Primer sequence (5' to3') \\
\hline IncTUG1 & Forward: 5'-CTGAAGAAAGGCAACATC-3' \\
miR-144-3p & Reverse: 5'-GTAGGCTACTACAGGATTTG-3' \\
& Forward: 5'- CCCTACAGTATAGATGATG -3' \\
& Reverse: 5'-TGCAGGGTCCGAGGT-3' \\
c-Met & Forward: 5'-CATGCCGACAAGTGCAGTA-3' \\
& Reverse: 5'-TCTTGCCATCATTGTCCAAC-3' \\
GAPDH & Forward: 5'-ATCCACGGGAGAGCGACAT-3' \\
& Reverse: 5'-CAGCTGCTTGTAAAGTGGAC-3' \\
U6 & Forward: 5'-ACAGATCTGTCGGTGTGGCAC-3' \\
& Reverse: 5'-GGCCCCGGATTATCCGACATTC-3' \\
\hline
\end{tabular}

\section{Dual-luciferase reporter assays}

Luciferase reporter gene vectors (pRL-TK, Promega) containing wild type (WT) or mutant (Mut) lncTUG1 and the 3'-UTR of WT or Mut MET were transfected into HEK293T cells. The miR-144-3p mimic, miR-144$3 p$ inhibitor or negative control (NC) was cotransfected with reporter plasmids for $48 \mathrm{~h}$. Relative luciferase activity was determined using a Dual-Luciferase Reporter Assay System (Promega).

\section{Cell viability assays}

A total of 5000 cells were seeded into a 96-well plate for $24 \mathrm{~h}$, and then cells were exposed to $2 \mathrm{~Gy}$ radiation (once). After radiotherapy, cell viability was evaluated by the MTT assay at $0,24,48,72$ and $96 \mathrm{~h}$. A range of radiation doses $(0,2,4,6$ and 8 Gy) was applied in a dosedependent experiment.

\section{Colony formation assays}

Five hundred cells were seeded into a 6-well plate with or without $2 \mathrm{~Gy}$ radiation. After two weeks, the cells were fixed and stained with $0.1 \%$ crystal violet solution. The numbers of colonies were counted under an inverted microscope.

\section{Flow cytometry}

EC9706 and KYSE30 cells were harvested at $48 \mathrm{~h}$ posttransfection. An Annexin V-FITC/PI Apoptosis Detection Kit (Sigma-Aldrich, St. Louis, MO, USA) was utilized to detect cell apoptosis according to the manufacturer's instructions, and the percentage of apoptotic cells was calculated using a Beckman Coulter FACS flow cytometer (Beckman Coulter).

\section{Western blot analysis}

The cells were lysed in RIPA buffer (Sigma-Aldrich). After centrifugation, the protein was extracted, and the concentration was quantified using a BCA assay (Pierce, Rockford, IL, USA). Then, protein samples were separated by $10 \%$ SDS-PAGE and transferred onto polyvinylidene fluoride (PVDF) membranes (Amersham Pharmacia, Little Chalfont, UK). The primary antibodies used were anti-cMET (1:1000, Thermo Fisher Scientific), anti-EGFR (1: 2500, Invitrogen), anti-t-AKT (1:2000, Cell Signaling), antip-AKT (1:500, Invitrogen), and anti-GAPDH (1:1000, Invitrogen), and a secondary horseradish peroxidase (HRP)conjugated antibody (Invitrogen) was used. GAPDH was chosen as the internal loading control.

\section{RNA immunoprecipitation (RIP) assays}

A Magna RIP ${ }^{\mathrm{Tw}}$ RNA-Binding Protein Immunoprecipitation Kit (Millipore, USA) was used for RIP experiments according to the manufacturer's instructions. The TUG1 level was detected by qRT-PCR. 


\section{Xenograft mouse model}

Twenty male BALB/c nude mice (age, 6 weeks; sex, male; weight, $20 \mathrm{~g}$ ) were obtained by the Cancer Hospital of the CAMS and maintained in a pathogen-free animal facility at $24{ }^{\circ} \mathrm{C}$ with access to distilled food and water. A total of $3 \times$ $10^{6}$ transfected (LV-NC or LV-TUG1) KYSE30 cells were subcutaneously injected into six-week-old male nude mice ( $n=5$ per group). The mice were given radiation ( 2 Gy) for 5 consecutive days when the tumors reached an average volume of approximately $100 \mathrm{~mm}^{3}$. Tumor volume was measured every three days according to the following formula: volume $=1 / 2 \times$ length $\times$ width $^{2}$. All animal procedures were performed following approval from the Animal Care and Use Committee of the Cancer Hospital of the CAMS.

\section{Immunohistochemistry}

All tissues were cut into $4-\mu \mathrm{m}$ sections. The sections were incubated with an anti-Ki67 antibody (1:200, Abcam, Cambridge, UK), MET antibody (1:200, GeneTex, GTX50668) and p-AKT antibody (1:200, GeneTex, GTX128414) at $4{ }^{\circ} \mathrm{C}$ overnight. Then, biotinylated secondary antibodies were incubated for $1 \mathrm{~h}$ at room temperature and visualized with diaminobenzidine substrate (Sigma-Aldrich, St. Louis, MO, USA). Immunohistochemistry (IHC) images were taken using an Olympus microscope.

\section{Statistical analysis}

Statistical analysis was performed using SPSS 19.0 software (SPSS, Chicago, IL, USA). The data are expressed as the mean \pm standard deviation (SD). Differences between groups were evaluated by Student's t-test or oneway analysis of variance (ANOVA). $P<0.05$ indicated statistical significance.

\section{Results}

\section{Bioinformatics analysis shows that IncTUG1 might} participate in ESCC

To identify candidate genes that are associated with ESCC radioresistance, we performed a bioinformatics analysis using published expression data (Fig. 1a). Briefly, two data series consisting of two esophageal cancer cells and their derived radioresistant cell lines were obtained from the Gene Expression Omnibus (GEO) database (i.e., GSE61620, and GSE61772). Differential expression analysis was then performed between radioresistant and radiosensitive cell lines under different irradiation conditions using the normalized microarray data, which identified 341 genes that were significantly upregulated and 594 genes that were significantly downregulated in the radiosensitive cell lines compared with the radioresistant cell lines $(P<0.05$; Fig. 1b). As shown in Fig. 1c and d, lncRNA-TUG1 was one of the most upregulated molecules, suggesting that it might play a role in the development of radiotherapy resistance in ESCC. To investigate the biological function of lncRNA-TUG1, genes whose expression levels were tightly correlated (absolute Pearson's correlation coefficient value $>0.9$ ) with that of the molecule in the cell lines were selected as the input for Metascape pathway analysis [26]. These genes were significantly enriched in meaningful cancer-related processes or pathways, such as the 'Cell Cycle' and 'Transcriptional Regulation by TP53' (Fig. 1e). To facilitate illustration, Circos was used to visualize the genes related to lncRNA-TUG1 expression in the GO0044772 term (Fig. 1f). To further explore the potential mechanism of lncTUG1 in radioresistance, RAID v2.0 was used to identify molecules that interact with lncTUG1 [27]. Indeed, we observed that hsa-miR-144-3p and hsa-miR145-5p achieved the highest confidence scores among all types of interactors. The target prediction information is shown in Fig. 1g. Below, we focus on the relationship between lncTUG1 and hsa-miR-144-3p.

IncTUG1 is upregulated in both ESCC tissues and cell lines Based on the above results, we first examined the expression levels of lncTUG1 and its potential interacting miRNA, miR-144-3p, in ESCC and paired normal tissues. As shown in Fig. 2a-d, lncTUG1 was highly expressed in tumor tissues, while miR-144-3p was weakly expressed. In line with this result, the expression of lncTUG1 was increased in ESCC cell lines compared with normal esophageal cell lines (Fig. 2e). Moreover, decreased miR-144-3p expression was also observed in all the analyzed ESCC cell lines (Fig. 2f). Pearson's correlation analysis confirmed that the expression of lncTUG1 was inversely correlated with that of miR-144-3p in both tissues and cell lines (Fig. 2g).

\section{IncTUG1 knockdown inhibits cell proliferation, migration and invasion}

To investigate the functional role of lncTUG1 in tumorigenesis, we silenced lncTUG1 expression in EC9706 and KYSE30 cells with an siRNA. As shown in Fig. 3a, siTUG1 was successfully transfected into the cell lines, and endogenous lncTUG1 was significantly suppressed. Then, the relative cellular abilities of proliferation, migration and invasion were examined. si-TUG1 retarded the growth of EC9706 and KYSE30 cells according to the MTT assay (Fig. 3b and c), and the results of the colony formation assay were largely consistent (Fig. 3d). Moreover, the low level of lncTUG1 expression led to a downward trend in both migration and invasion (Fig. 3e and f). In contrast, lncTUG1 knockdown increased the quantity of apoptotic cells (Fig. 3g). Taken together, these results indicate 
A

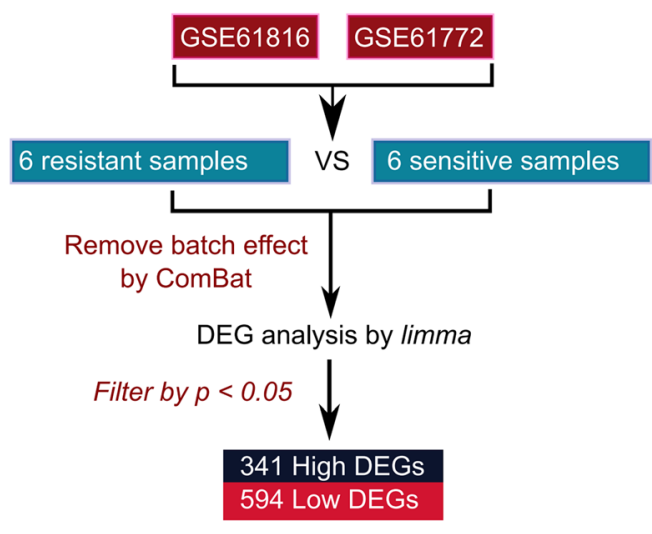

C

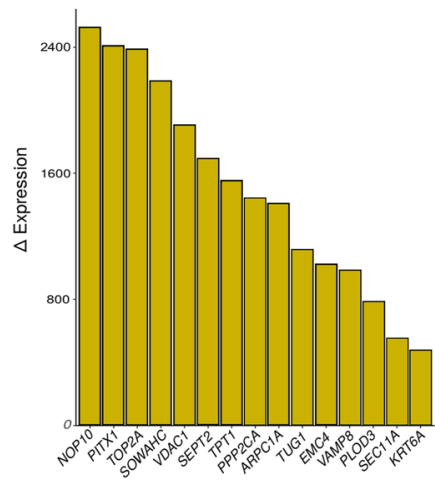

E

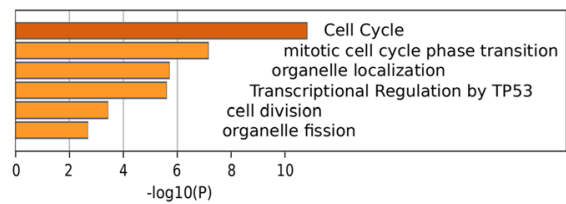

B

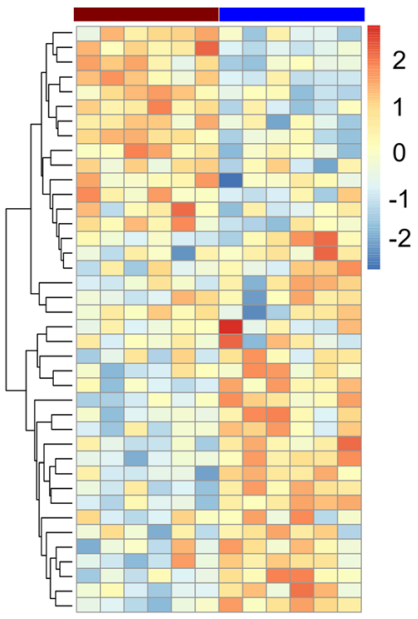

$\mathrm{F}$
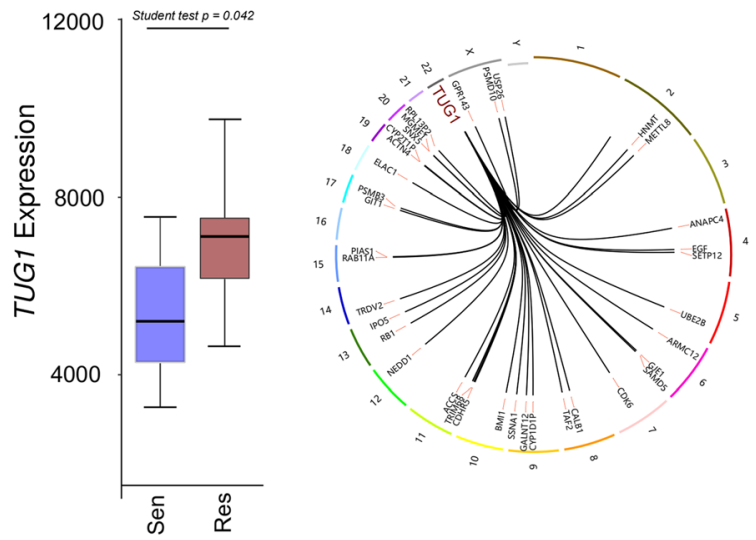

G

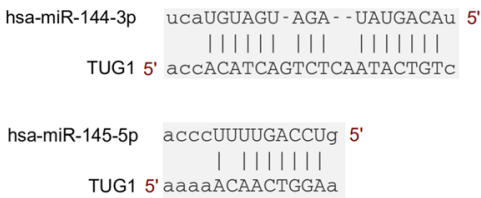

TUG1 5' aaaaACAACTGGAa

Fig. 1 Bioinformatics analysis shows that IncTUG1 might participate in ESCC (a). The basic flow of data mining analysis; (b). The genes with significantly different expression are shown as a heat map, relative enrichment scores indicate upregulated (red) and downregulated (blue) genes across samples; (c). The top 15 genes with significantly higher expression in radioresistant cell lines; (d). The expression level of IncTUG1 in sensitive and resistant samples; (e). Pearson's correlation of IncTUG1 and cell processes; (f). The Circos figure shows the genes from GO0044772 which are significantly correlated with IncTUG1; (g). The binding site between IncTUG1 and its potential interactors

that lncTUG1 is a potential oncogenic factor that influences the progression of ESCC.

\section{IncTUG1 is involved in ESCC radiotherapy and affects radiosensitivity}

Because the analysis was performed in the radiotherapy samples, the mechanism by which lncTUG1 affects the radiosensitivity of ESCC cells was the point of interest.
The level of lncTUG1 was examined in a time- and dose-dependent manner in EC9706 and KYSE30 cells. Both the dose and time affected the expression level of lncTUG1 ( $" P<0.05$, Fig. 4a and b). More importantly, siTUG1 combined with 2 Gy radiation showed increased radiation sensitivity in ESCC cells. The MTT assay indicated that this combined treatment had significant inhibitory effects on cell proliferation $(* P<0.05$, Fig. 4c). 


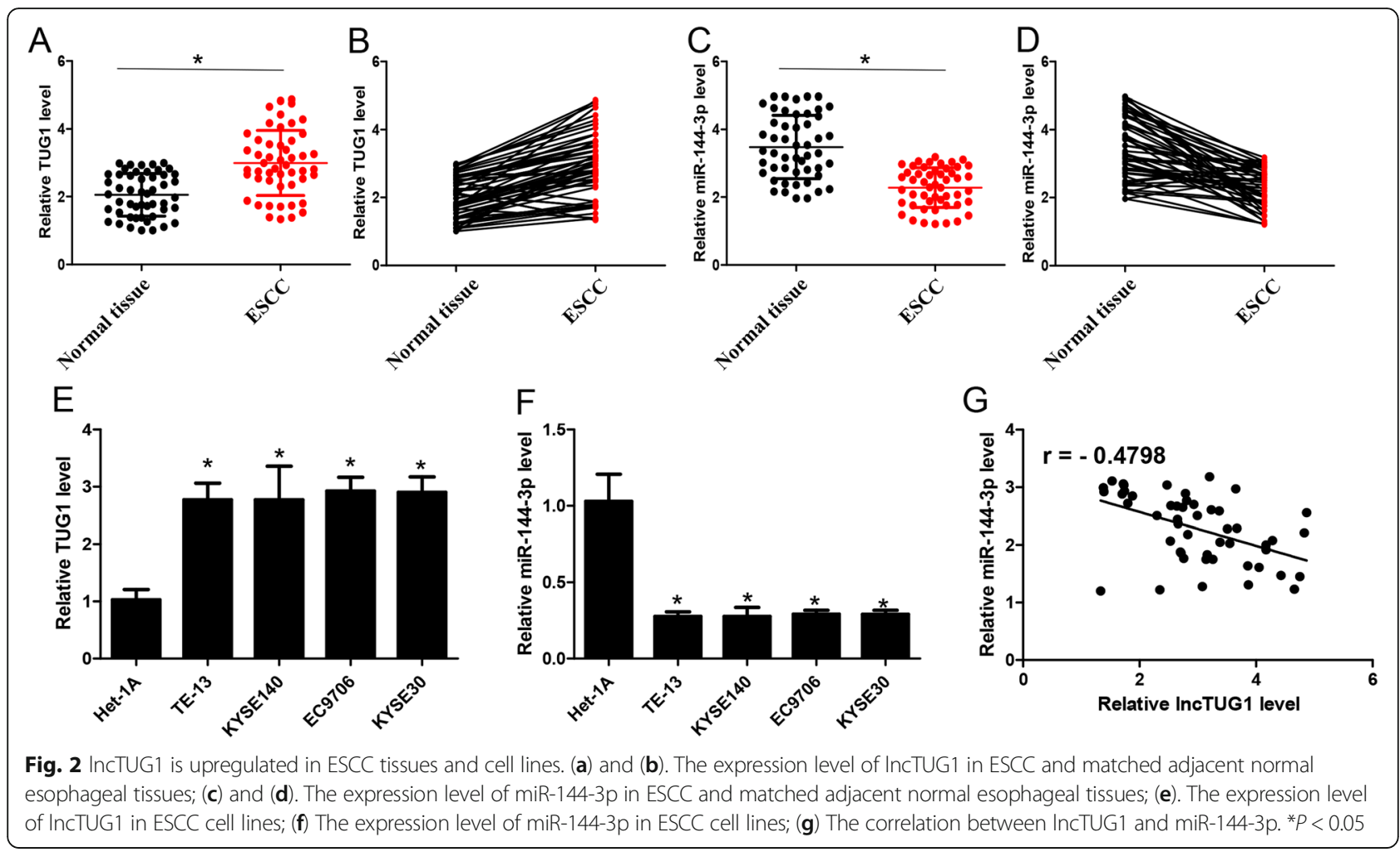

EC9706 and KYSE30 cell colonies were inhibited dramatically by lncTUG1 knockdown plus 2 Gy radiation (Fig. 4d). Moreover, this combined treatment further induced the apoptosis of EC9706 and KYSE30 cells $\left({ }^{*} P<0.05\right.$, Fig. 4e). Besides these results, in Fig. 4f, we conducted the colony formation assay of EC9706 and KYSE30 cells exposed to the different radiotherapy doses $(0,2,4,6,8 \mathrm{~Gy})$. As shown in Fig. 4g, the cellular survival curves of EC9706 and KYSE30 cells indicated the knockdown of lncTUG1 indeed enhance the radiosensitivity, and the relative relative radiosensitizaion effects data were shown in Table 3. All these results indicate that lncTUG1 is involved in ESCC radiotherapy and affects radiosensitivity.

\section{IncTUG1 affects ESCC progression via the miR-144-3p/MET axis}

miR-144-3p was chosen as a target based on the previous prediction. The luciferase system indicated that only the miR-144-3p mimic decreased the luciferase activity of WT-TUG1 but had no effect on MutTUG1 (Fig. 5a). The lncTUG1 level was affected by the miR-144-3p level (Fig. 5b). Moreover, the RIP assay further confirmed that lncTUG1 was significantly augmented by Ago2 but not IgG (Fig. 5c). To further identify the target of hsa-miR-144-3p, the 3 'UTR of MET with the potential binding site was examined (Fig. 5d). The luciferase reporter system showed that the luciferase activity of the 3 '-UTR of only WT MET was decreased (Fig. 5e). Both the protein and mRNA levels of MET were affected by the miR-144-3p level (Fig. 5f and g). Moreover, the miR144-3p inhibitor reversed the si-TUG1 effect on the protein level of MET (Fig. 5h). Based on these results, we conclude that the lncTUG1/ miR-144-3p/MET axis indeed exists.

The miR-144-3p inhibitor restores the effect of IncTUG1 knockdown on radiotherapy

According to the above results, lncTUG1 affects ESCC progression via the miR-144-3p/MET axis. We further determined whether lncTUG1 affects radiosensitivity through miR-144-3p and MET. As shown in Fig. $6 a$ and b, colony formation and apoptosis assays confirmed that the miR-144-3p inhibitor restored the effect of lncTUG1 knockdown on radiotherapy. Moreover, MET knockdown decreased the level of EGFR and lowered the phosphorylation level of AKT (Fig. 6c). It is possible that the p-AKT level is the key factor in ESCC radiotherapy.

In vivo experiments confirmed that the inhibition of IncTUG1 enhances ESCC radiosensitivity

Finally, we aimed to explore the effect of lncTUG1 on the radiosensitivity of ESCC tumor tissue. We subcutaneously injected transfected (LV-NC or LV- 


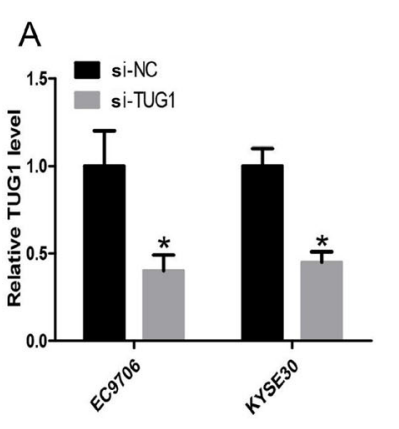

$\mathrm{D}$

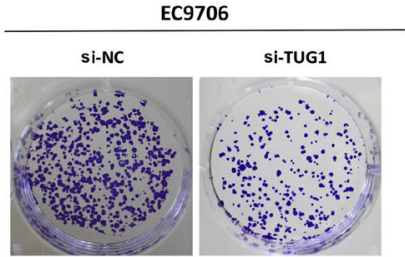

E

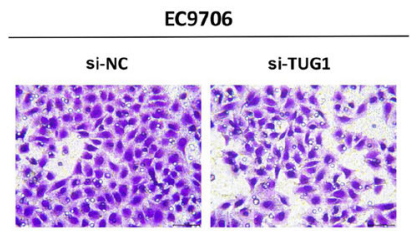

$\mathrm{F}$

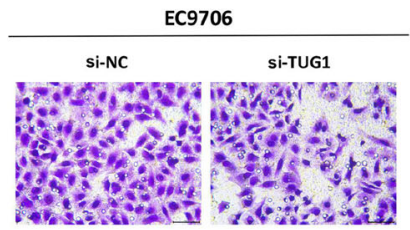

G

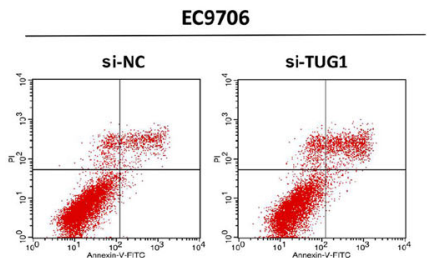

B

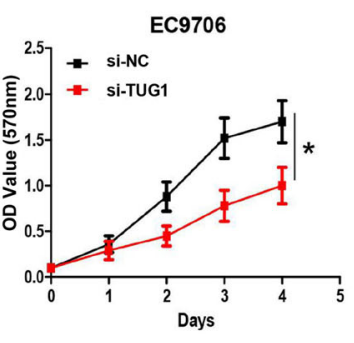

C

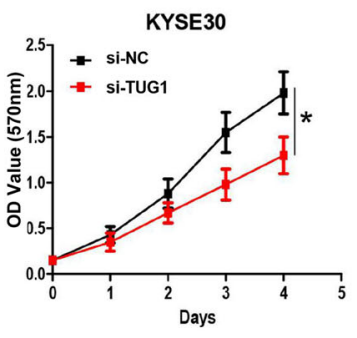

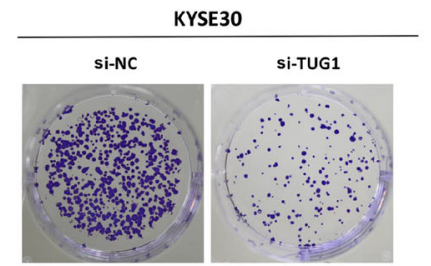
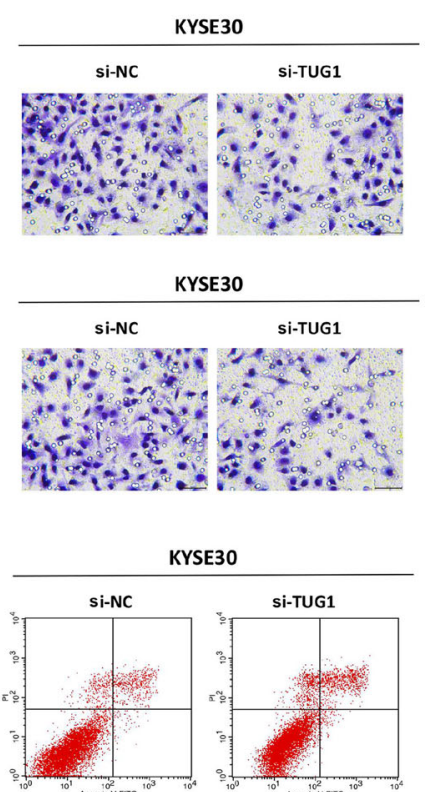
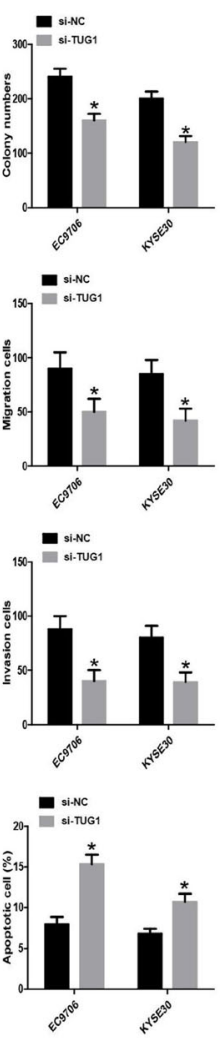

Fig. 3 IncTUG1 knockdown inhibits ESCC cell proliferation, migration and invasion. a The level of IncTUG1 in EC9706 and KYSE30 cells; (b) and (c). Cell proliferation was evaluated with the MTT assay; (d). Cell proliferation was evaluated with the colony formation assay; (e) and (f). The migration and invasion abilities of EC9706 and KYSE30 cells; (g). Cell apoptosis was evaluated by flow cytometry. ${ }^{*} P<0.05$

TUG1) KYSE30 cells into BALB/c nude mice to establish an in vivo model. The findings indicated that lncTUG1 knockdown could enhance the effect of radiotherapy on ESCC in vivo (Fig. 7a, b and c). All of these results exhibited the smallest tumor volume, the slowest tumor growth and the lightest tumor weight when the LV-TUG1 KYSE30 underwent 2 Gy radiation in this xenograft model. Meanwhile we also detected the downstream target expression. The tumor level of Ki67 was also reduced dramatically in the sh-TUG1 plus 2 Gy group (Fig. $7 \mathrm{~d}$ ). The tumor level of MET and p-AKT had the same trend that the lowest level of MET and p-AKT in the sh-TUG1 plus 2 Gy group (Fig. 7e and f).

\section{Discussion}

In this study, we discovered that lncTUG1, as an oncogenic factor, participates in the progression of ESCC. More importantly, the role of lncTUG1 in the radiosensitivity of ESCC was investigated. Our findings revealed that lncTUG1 increases the expression of MET by sponging miR-144-3p and then activates the AKT signaling pathway to promote the progression of ESCC, including inhibiting apoptosis and 

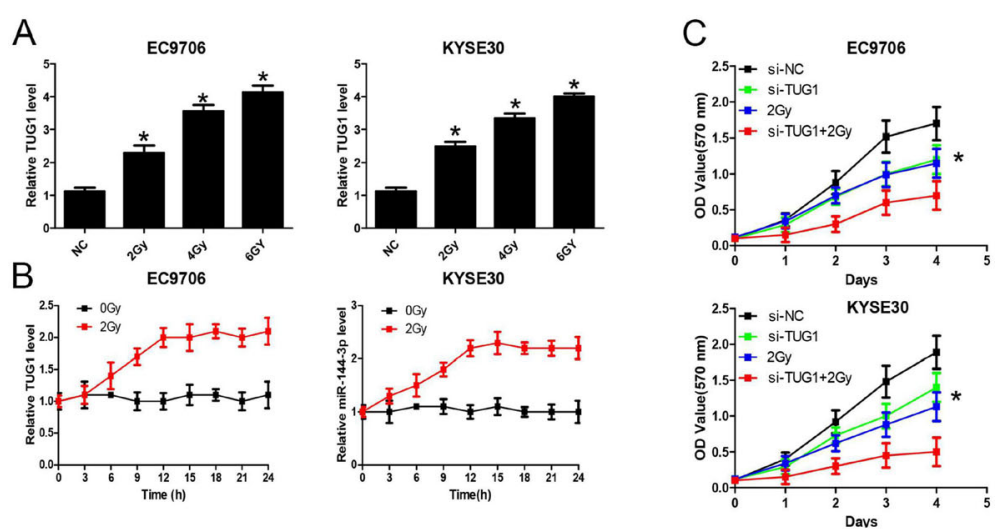

\section{D}

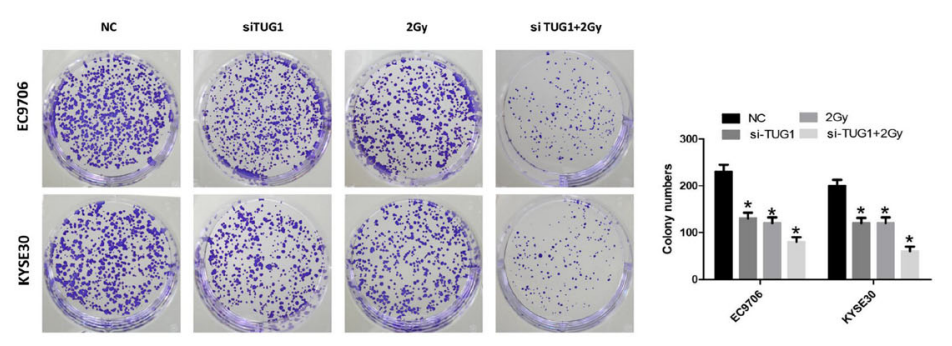

$\mathrm{E}$

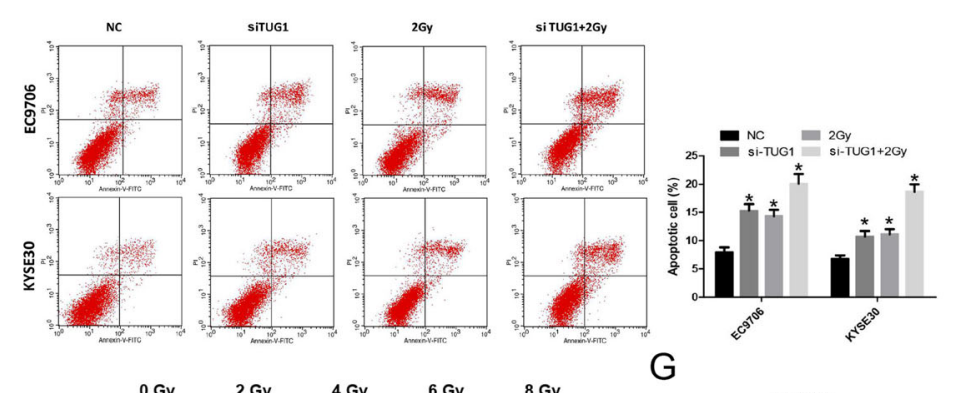

$\mathrm{F}$
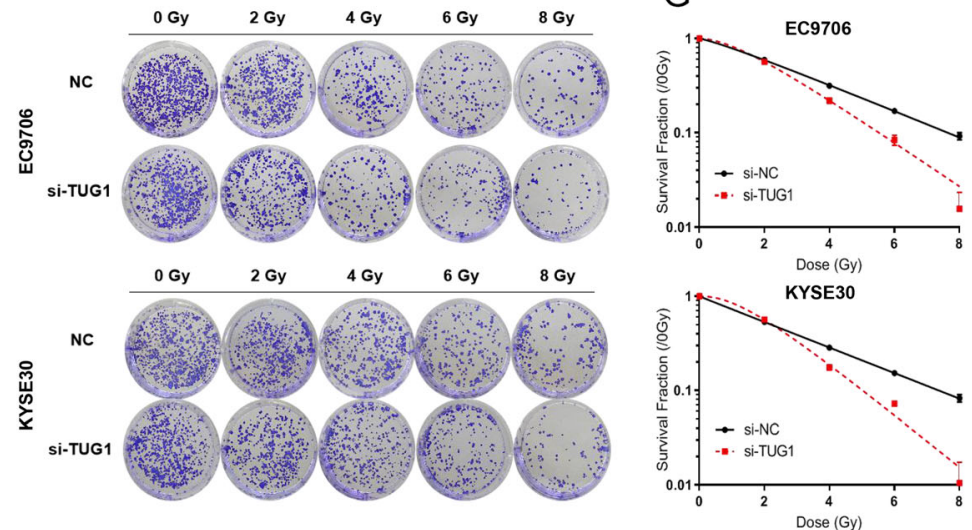

Fig. 4 IncTUG1 is involved in ESCC radiotherapy and affects radiosensitivity. a and b. The level of IncTUG1 in EC9706 and KYSE30 cells; (c) and (d). Cell proliferation was evaluated with MTT and colony formation assays; (e). Cell apoptosis was evaluated by flow cytometry; (f). The colony forming ability of EC9706 and KYSE30 cells were exposed to 0, 2, 4, 6, 8 Gy; (g). the cellular survival curves of EC9706 and KYSE30 cells. ${ }^{*} P<0.05$

inducing proliferation, migration and invasion. These results were consistent with those of previous reports that indicated that lncTUG1 might be an oncogenic factor. For example, Li Y et al. found that lncTUG1 was upregulated in renal cell carcinoma and acted as a miR-299-3p sponge to promote tumorigenesis by activating the VEGF pathway [28]. $\mathrm{Xu} \mathrm{T}$ et al. also reported that lncTUG1 accelerated prostate cancer 
Table 3 The relative radiosensitizaion effects in EC9706 and KYSE30

\begin{tabular}{cllll}
\hline & D0 (Gy) & Dq (Gy) & $\mathrm{N}$ & $\mathrm{SF}_{2}(\%)$ \\
\hline EC9706 & & & & \\
Si-NC & $3.073 \pm 0.124$ & $1.475 \pm 0.079$ & $1.616 \pm 0.032$ & $69.6 \pm 1.0$ \\
Si-TUG1 & $1.865 \pm 0.110^{*}$ & $1.297 \pm 0.042$ & $2.005 \pm 0.093^{*}$ & $56.8 \pm 0.9$ \\
KYSE30 & & & & \\
Si-NC & $3.209 \pm 0.123$ & $2.215 \pm 0.124$ & $1.994 \pm 0.023$ & $78.3 \pm 0.9$ \\
Si-TUG1 & $1.578 \pm 0.114^{*}$ & $1.463 \pm 0.039$ & $2.528 \pm 0.155^{*}$ & $56.7 \pm 0.8$ \\
\hline${ }^{*}<<0.05$ & & & &
\end{tabular}

tumorigenesis and was associated with a poor prognosis [29]. Based on our findings, lncTUG1 promotes proliferation, migration and invasion but inhibits apoptosis in ESCC cells. In summary, we believe that lncTUG1 should serve as an oncogenic factor in the development of ESCC.

miR-144-3p and MET were found to affect the development of ESCC. Moreover, we verified that miR144-3p can downregulate the expression of MET by the dual-luciferase reporter system. Mushtaq et al. reported that miR-144 exhibited tumor suppressive effects on gastric cancer cells [30]. It was reported that a high level of miR-144, as a promising therapeutic strategy, alleviated resistance to chemotherapy in glioblastoma cells [31]. miR-144-3p can inhibit the SrcAkt-Erk pathway to retard the progression of lung cancer [32]. Moreover, numerous studies have indicated that MET is associated with activation of the AKT signaling pathway by upregulating the expression level of EGFR. MET/EGFR signaling modulates cell proliferation in lung cancer [33]. The biological roles of these factors are consistent with our findings; thus, we provide new insights into the oncogenic role of lncTUG1, which promotes the development of ESCC through the miR-144-3p/MET/AKT axis.

We also note that there are some limitations to our study. Both lncTUG1 and miR-144-3p could have additional targets needed to exert their biological functions. They may play important roles in ESCC through multilevel regulation, leading to synthetic effects. By high-throughput sequencing analysis, the underlying
A

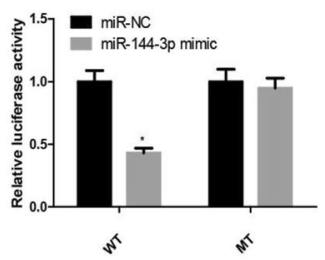

D

MET 3'-UTR 5' ...GAAUUUUGUGCUUGCUACUGUAU...
$\begin{aligned} & \text { 1430-1436 } \\ & \text { hsa-miR-144-3p 3' } \quad \text { UCAUGUAGUAGAUAUGACAU }\end{aligned}$

E

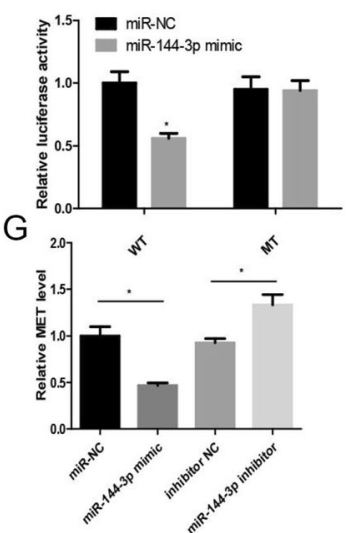

B
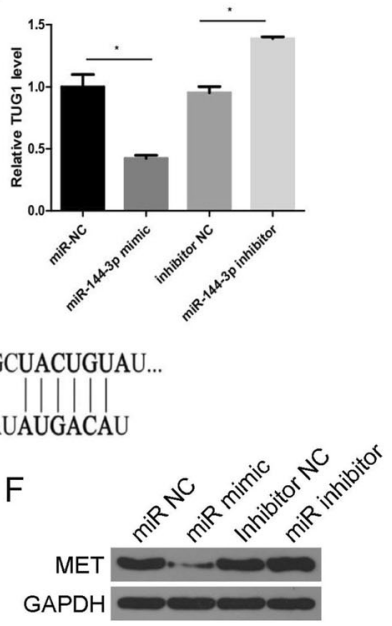

$\mathrm{H}$

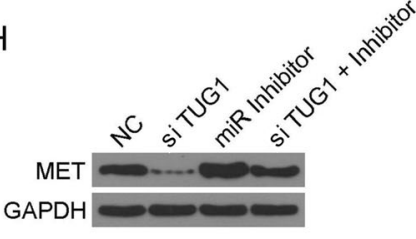

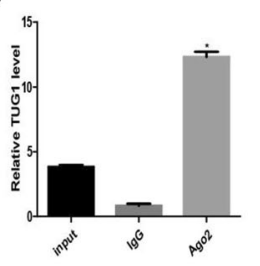

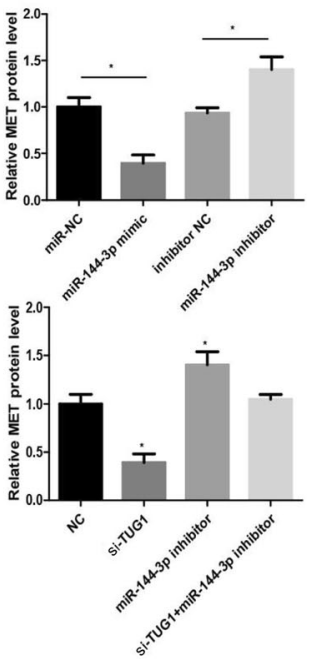

Fig. 5 IncTUG1 affects ESCC progression via the miR-144-3p/MET axis. a. Luciferase activity was examined in HEK293T cells; (b). The level of IncTUG1 in KYSE30 cells; (c). The interaction between miR-144-3p and LncTUG1 was detected by RNA immunoprecipitation; (d). The potential binding site between miR-144-3p and MET; (e). Luciferase activity was examined in HEK293T cells; (f). MET protein level in KYSE30 cells; (g). MET mRNA level in KYSE30 cells; (h). MET protein level in KYSE30 cells. * $P<0.05$ 
A

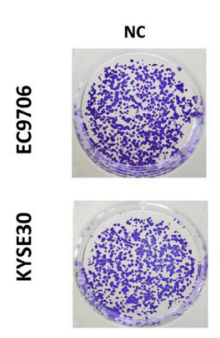

B

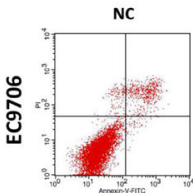

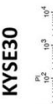

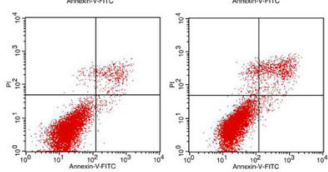

C

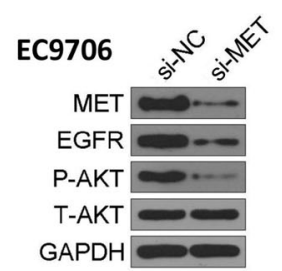

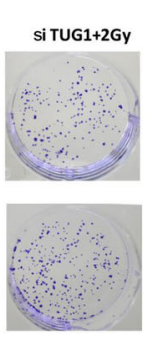

si TUG1+2Gy
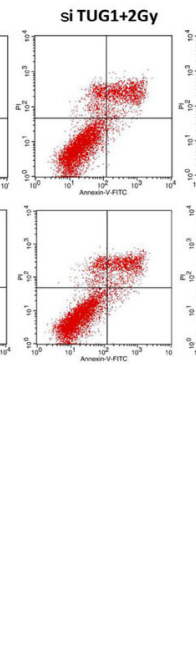

si TUG1+2Gy

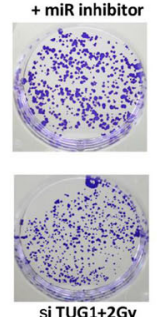

Si TUG1+2Gy
+ miR inhibitor
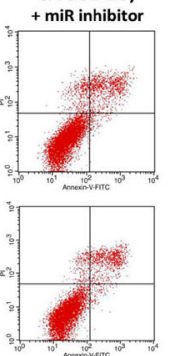

KYSE30
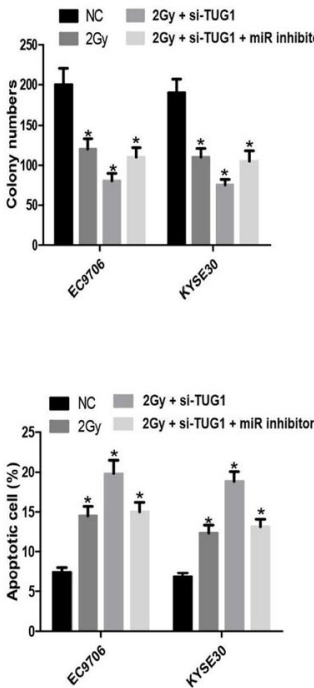

20

MET

EGFR

P-AKT

T-AKT

GAPDH
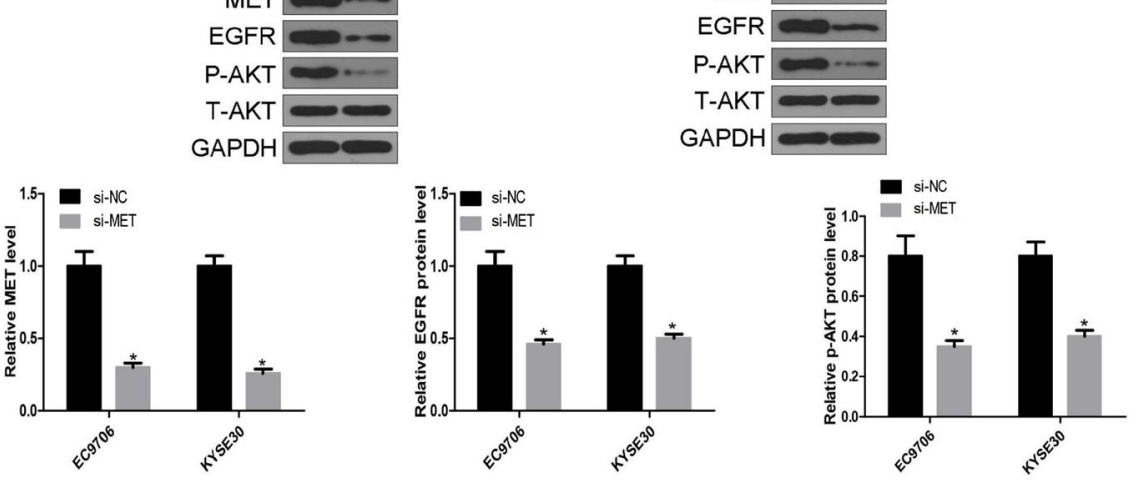

…

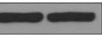
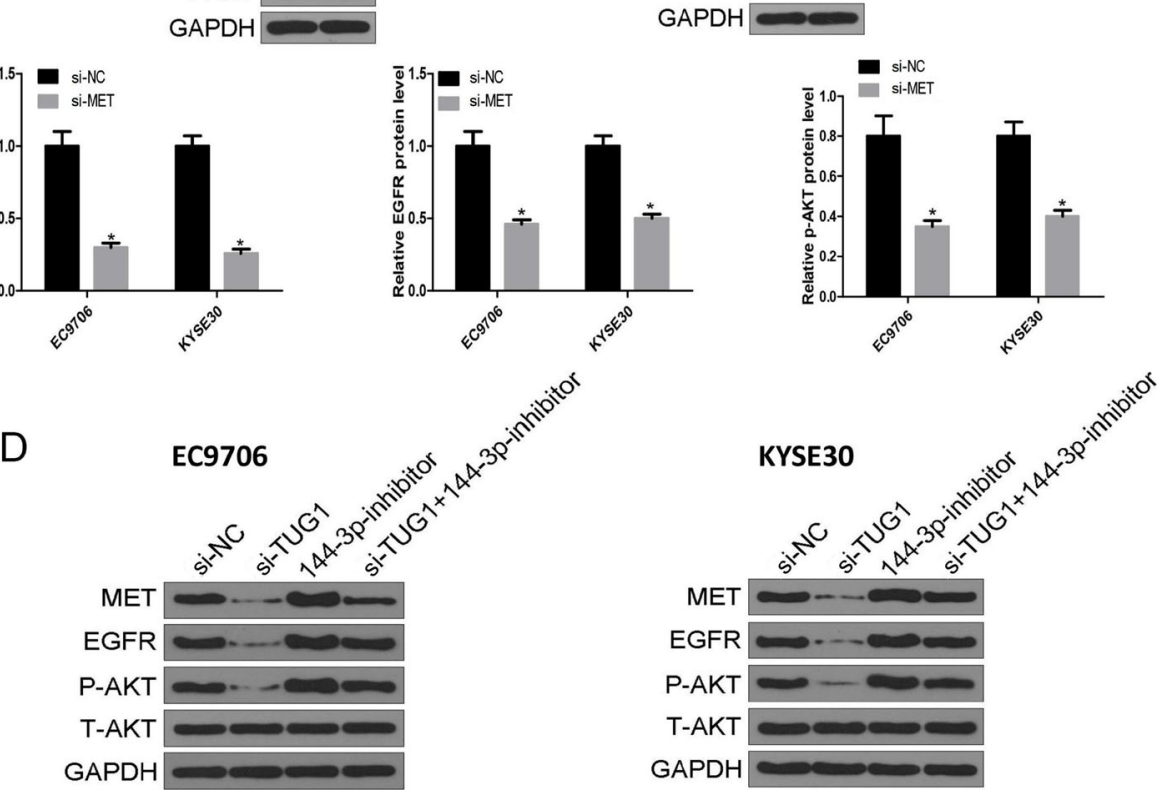

Fig. 6 The miR-144-3p inhibitor restores the effect of IncTUG1 knockdown on radiotherapy. a Cell proliferation was evaluated by colony formation assays; (b). Cell apoptosis was evaluated by flow cytometry; (c) and (d). The protein levels of MET, p-AKT and t-AKT in EC9706 and KYSE30 cells. ${ }^{*} P<0.05$

biological changes in the different expression levels of lncTUG1 will be uncovered.

More importantly, we are highly concerned with improving the effect of radiotherapy on ESCC. First, by analyzing expression information on ESCC tissues and radiotherapy samples from the GEO database, we found an apparent difference in lncTUG1 between sensitive and resistant samples. Second, in combination with 2 Gy radiotherapy, we verified that lncTUG1 affected the progression of ESCC in vivo and in vitro. This result suggests that lncTUG1 regulates radiosensitivity in ESCC. Third, the phosphorylation of AKT, as a key 
A

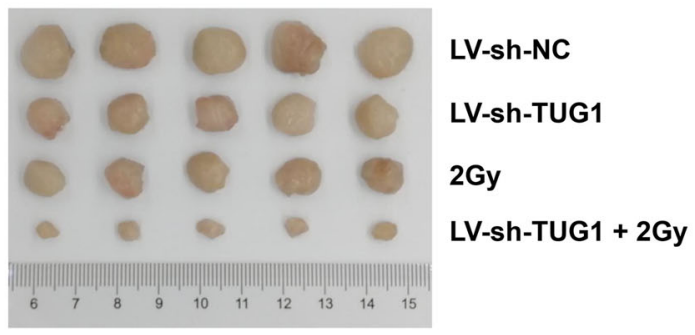

B

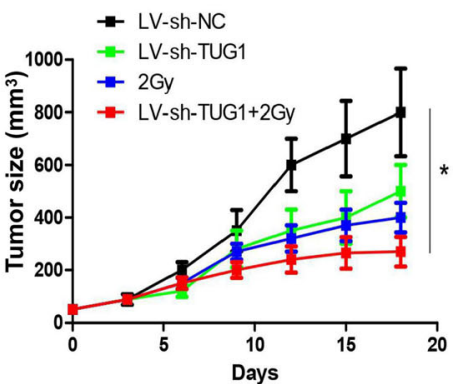

C

D

The levels of Ki67
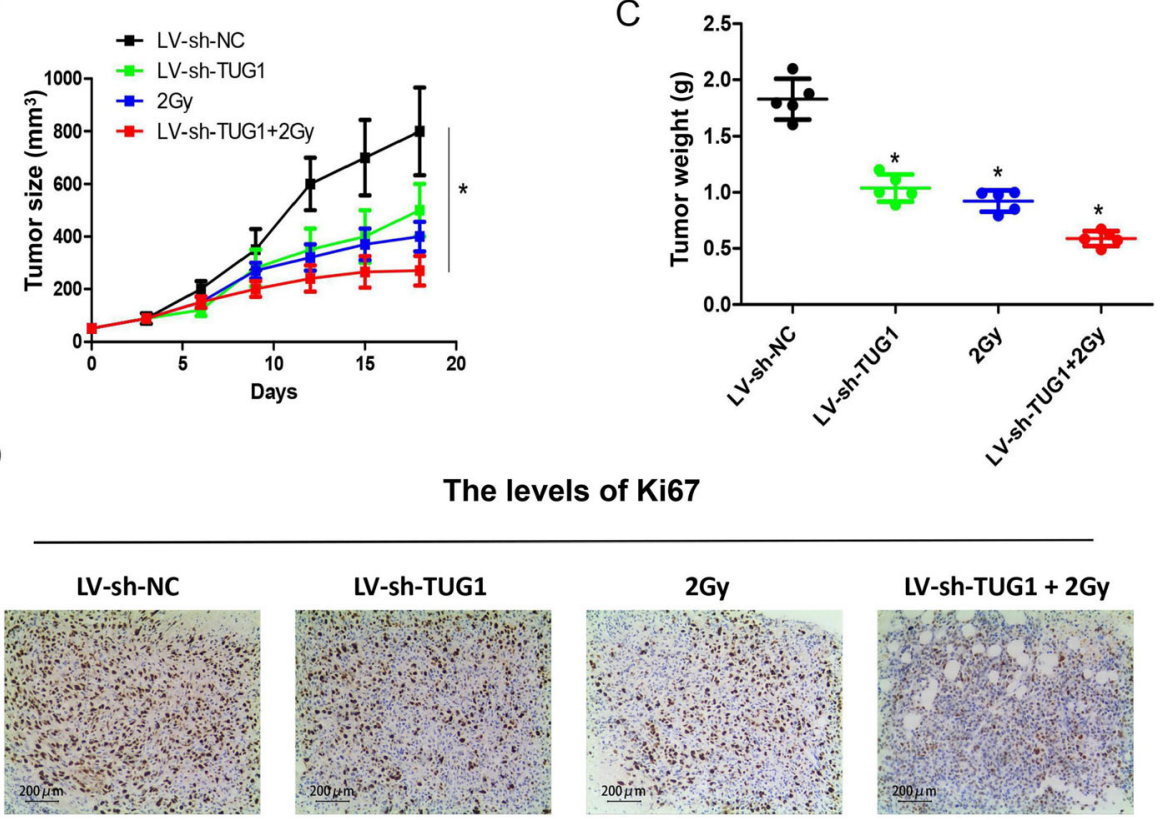

$\mathrm{E}$

The levels of MET

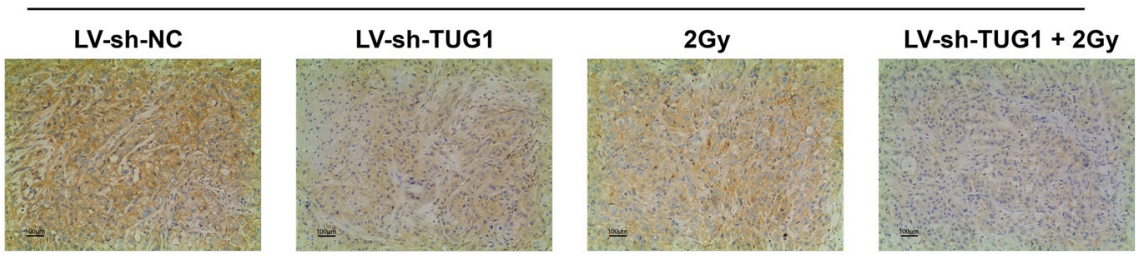

$\mathrm{F}$

The levels of $\mathrm{p}-\mathrm{AKT}$

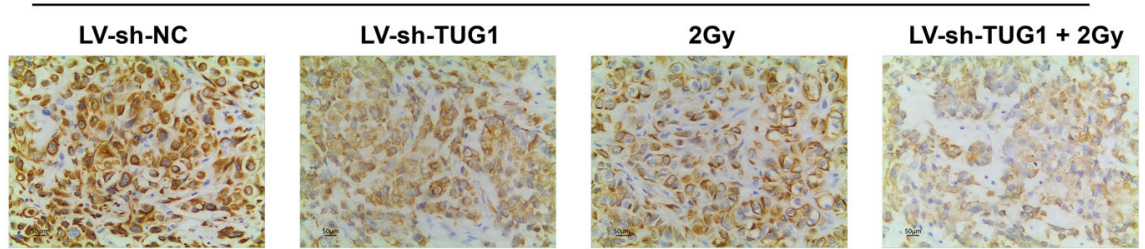

Fig. 7 In vivo experiments confirmed that the inhibition of IncTUG1 enhances ESCC radiosensitivity. a Tumor images; (b) Tumor growth curves; (c). Tumor weights; (d), (e) and (f) Ki67, MET and p-AKT tumor levels based on IHC. ${ }^{*} P<0.05$

factor related to radiosensitivity, is influenced by the level of lncTUG1. Notably, lncTUG1 exerts an apparent radiotherapy resistant effect on ESCC. Thus, lncTUG1 knockdown potentially has significant clinical application value.

\section{Conclusions}

In conclusion, our study clarifies that lncTUG1 elevates the expression of MET by sponging miR-144-3p and then activates the AKT signaling pathway to affect the radiosensitivity of ESCC. 


\section{Acknowledgements}

Not applicable.

\section{Authors' contributions}

All authors contributed to data analysis, drafting or revising the article, gave final approval of the version to be published, and agree to be accountable for all aspects of the work. All authors have read and approved the final manuscript.

\section{Funding}

This work was supported by the National Natural Science Foundation of China [81802299]; the Chinese Academy of Medical Sciences Innovation Fund for Medical Sciences [2016-12M-1-001, 2017-12M-1-005]; the National Key Basic Research Development Plan [2018YFC1312105]; and the Beijing Municipal Science \& Technology Commission [Z181100001918002].

\section{Availability of data and materials}

The analyzed data sets generated during the study are available from the corresponding author on reasonable request.

\section{Ethics approval and consent to participate}

The present study was approved by the Ethics Committee of Cancer Hospital of the Chinese Academy of Medical Sciences. The research has been carried out in accordance with the World Medical Association Declaration of Helsinki. All patients and healthy volunteers provided written informed consent prior to their inclusion within the study.

All animal procedures were performed following approval from the Animal Care and Use Committee of the Cancer Hospital of the CAMS.

\section{Consent for publication}

Not applicable.

\section{Competing interests}

The authors declare that they have no competing interests.

\section{Author details}

'Department of Thoracic Surgery, National Cancer Center/National Clinica Research Center for Cancer/Cancer Hospital, Chinese Academy of Medical Sciences and Peking Union Medical College, No.17 Panjiayuannanli, Beijing 100021, China. 'Department of Radiation Oncology, National Cancer Center/ National Clinical Research Center for Cancer/Cancer Hospital, Chinese Academy of Medical Sciences and Peking Union Medical College, Beijing 100021, China. ${ }^{3}$ Cancer Institute, The Affiliated Hospital of Qingdao University, Qingdao 266071, Shandong, China. ${ }^{4}$ Qingdao Cancer Institute, Qingdao 266071, Shandong, China.

\section{Received: 23 October 2019 Accepted: 30 December 2019}

Published online: 09 January 2020

\section{References}

1. $F B, J F, I S, R L S, L A T, A$ J: Global cancer statistics 2018: Globocan estimates of incidence and mortality worldwide for 36 cancers in 185 countries. CA Cancer J Clin 2018;68:394-424.

2. L DC, W MR, K HP. Genomic and epigenomic aberrations in esophageal squamous cell carcinoma and implications for patients. Gastroenterol. 2018; 154:374-89.

3. C CS, L YC, W MS. Prevention strategies for esophageal cancer: perspectives of the east vs. west. Best Pract Res Clin Gastroenterol. 2015;29:869-83.

4. $\quad P A, G M K, J B A, L J D$. Oesophageal carcinoma. Lancet (London, England). 2013;381:400-12

5. N K, S M, M S. esophageal cancer: a review of epidemiology, pathogenesis, staging workup and treatment modalities. World J Gastrointest Oncol. 2014; 6:112-20.

6. L J, S E, C D, L P. Oesophageal cancer. Lancet (London, England). 2017;390: 2383-96.

7. H J, S ME, C A, R FZ, S PD, vH R. A patient with tracheoesophageal fistula and esophageal cancer after radiotherapy. Nat Rev Gastroenterol Hepatol. 2010;7:702-6.

8. L C, Y L. Long noncoding rna in cancer: Wiring signaling circuitry. Trends Cell Biol. 2018;28:287-301.

9. Li J, Chen Z, Tian L, Zhou C, He MY, Gao Y, Wang S, Zhou F, Shi S, Feng X, Sun N, Liu Z, Skogerboe G, Dong J, Yao R, Zhao Y, Sun J, Zhang B, Yu Y, Shi
X, Luo M, Shao K, Li N, Qiu B, Tan F, Chen R, He J. Lncrna profile study reveals a three-Incrna signature associated with the survival of patients with oesophageal squamous cell carcinoma. Gut. 2014.

10. $Y L, F J E, L$ JT. Long noncoding rnas: Fresh perspectives into the rna world. Trends Biochem Sci. 2014;39:35-43.

11. $H M D, C W M, Q F Z, S M, X T P, M P, S Y Q$. long non-coding rna tug1 is upregulated in hepatocellular carcinoma and promotes cell growth and apoptosis by epigenetically silencing of klf2. Mol Cancer. 2015;14:165.

12. $X Y, W J, Q M, X L, L M, J F, Y R, X L$. Upregulation of the long noncoding rna tug1 promotes proliferation and migration of esophageal squamous cell carcinoma. Tumour Biol. 2015;36:1643-51.

13. J Z, S C, B Y, W M, X Y, J C: Molecular mechanisms of Incrnas in regulating cancer cell radiosensitivity. Biosci Rep 2019; undefined:undefined.

14. $L A M, Z Y, H Z W, L L, F S Z, C Y$. long noncoding rna fam201a involves in radioresistance of non-small-cell lung cancer by enhancing egfr expression via mir-370. Eur Rev Med Pharmacol Sci. 2019;23:5802-14.

15. SP K, V S, VK V, D A, P VJ, S C, K S: Cluster mirnas and cancer: Diagnostic, prognostic and therapeutic opportunities. Wiley Interdiscip Rev RNA 2019;undefined:e1563.

16. Bartel DP. Metazoan micrornas. Cell. 2018;173:20-51.

17. $L-U$ E, BM LP, LdGC D, P-P C, C-P AD. crosstalk between long non-coding rnas, micro-rnas and mrnas: deciphering molecular mechanisms of master regulators in cancer. Front Oncol. 2019;9:669.

18. G P, R T, V R, Z N. Mirnas in tumor radiation response: Bystanders or participants? Trends Mol Med. 2014;20:529-39.

19. $Y P, Y Y, A W, X J, Z G, J J, Z Q$. The long noncoding rna-ror promotes the resistance of radiotherapy for human colorectal cancer cells by targeting the p53/mir-145 pathway. J Gastroenterol Hepatol. 2017;32:837-45.

20. W J, Z Y, L F, Q B. Mir-144-3p: A novel tumor suppressor targeting mapk6 in cervical cancer. J Physiol Biochem. 2019;75:143-52.

21. C XY, S ZY, Z L, C MK. B Y: Microrna-144-3p suppresses human neuroblastoma cell proliferation by targeting hoxa7. Eur Rev Med Pharmacol Sci. 2019;23:716-23.

22. Organ SL, Tsao M-S. An overview of the c-met signaling pathway. Ther Adv Med Oncol. 2011;3:S7-S19.

23. S PK, S O. molecular dynamics guided development of indole based dual inhibitors of egfr (t790m) and c-met. Bioorg Chem. 2018;79:163-70.

24. S EJ, T BA, M D, F DM, D BR, W KJ, S IJ, I TM. Akt inhibition improves longterm tumour control following radiotherapy by altering the microenvironment. EMBO Mol Med. 2017;9:1646-59.

25. W Z, H Y, Z J. Molecularly targeting the pi3k-akt-mtor pathway can sensitize cancer cells to radiotherapy and chemotherapy. Cell Mol Biol Lett. 2014;19:233-42.

26. Zhou Y, Zhou B, Pache L, Chang M, Khodabakhshi AH, Tanaseichuk O, Benner C, Chanda SK. Metascape provides a biologist-oriented resource for the analysis of systems-level datasets. Nat Commun. 2019;10:1523.

27. Yi Y, Zhao Y, Li C, Zhang L, Huang H, Li Y, Liu L, Hou P, Cui T, Tan P, Hu Y, Zhang T, Huang Y, Li X, Yu J, Wang D. Raid v2.0: an updated resource of rnaassociated interactions across organisms. Nucleic Acids Res. 2017;45:D115-8.

28. $L Y, Z D, P L, D Y, C S, Z L, Z$ H. Knockdown of tug1 by shrna inhibited renal cell carcinoma formation by mir-299-3p/vegf axis in vitro and in vivo. Eur J Pharmacol. 2019:860:172536

29. $X T, L C L, L T, Z$ YH, Z YH. Lncrna tug1 aggravates the progression of prostate cancer and predicts the poor prognosis. Eur Rev Med Pharmacol Sci. 2019;23:4698-705

30. M F, Z J, L J. Mir-144 suppresses cell proliferation and invasion in gastric cancer through downregulation of activating enhancer-binding protein 4. Oncol Lett. 2019;17:5686-92.

31. AMS C, S M, M CM, O-C LR, R-V A, R O, T H, B M, P MCL, J AS. Mir-144 overexpression as a promising therapeutic strategy to overcome glioblastoma cell invasiveness and resistance to chemotherapy. Hum Mol Genet. 2019;28:2738-51.

32. $W J, Z X, L Y, J C, J Z, J Y$ : Microrna-144-3p suppressed tgf- $\beta 1$-induced lung cancer cell invasion and adhesion by regulating the src-akt-erk pathway. Cell Biol Int 2019; undefined:undefined.

33. Z J, F S, S W, B S, X L, W L, T DG, L J, R RM, C PW, L WR, C AC, B DG, G YM, $C G$. Overexpression of fam83h-as1 indicates poor patient survival and knockdown impairs cell proliferation and invasion via met/egfr signaling in lung cancer. Sci Rep. 2017;7:42819.

\section{Publisher's Note}

Springer Nature remains neutral with regard to jurisdictional claims in published maps and institutional affiliations. 